\title{
Supernova Polarization and the Type IIn Classification
}

\author{
Jennifer L. Hoffman \\ Department of Astronomy, UC Berkeley, 601 Campbell Hall, Berkeley, CA 94720-3411
}

\begin{abstract}
While the members of the Type IIn category of supernovae are united by the presence of strong multicomponent Balmer emission lines in their spectra, they are quite heterogeneous with respect to other properties such as Balmer line profiles, light curves, strength of radio emission, and intrinsic brightness. We are now beginning to see variety among SNe IIn in their polarimetric characteristics as well, some but not all of which may be due to inclination angle effects. The increasing number of known "hybrid" SNe with IIn-like emission lines suggests that circumstellar material may be more common around all types of $\mathrm{SNe}$ than previously thought. Investigation of the correlations between spectropolarimetric signatures and other IIn attributes will help us address the question of classification of "interacting SNe" and the possibility of distinguishing different groups within the diverse IIn subclass.
\end{abstract}

Keywords: supernovae, type IIn, supernova classification

PACS: $97.60 . \mathrm{Bw}$

Type IIn ("narrow-line") supernovae (SNe IIn) are Type II events whose primary distinction is the existence of strong narrow hydrogen Balmer emission lines in the spectra [1, 2]. These lines indicate the presence of circumstellar material ejected by the progenitor star and excited by the UV and X-ray photons from the supernova explosion. The IIn subclass includes 2-5\% of all Type II supernovae [3].

Turatto [4] categorized the SNe IIn as a special class of core-collapse supernovae related to the $\mathrm{SNe} \mathrm{Ib} / \mathrm{c}$ and hypernovae. However, in recent years several new discoveries have blurred the boundaries between the IIn category and other types of supernovae. It should be noted that since the label of IIn is assigned based on spectral characteristics, while that of II-L or II-P is based on features of the light curve, these three categories are not mutually exclusive, and some objects may be given more than one classification in the literature. But observations of "hybrid" supernovae such as SN 2002ic [5, 6] and SN 2005gj [7], which showed IIn-like $\mathrm{H} \alpha$ lines superposed on type Ia-like spectra, and of "chameleon" supernovae such as SN 2001em [8, 9], which evolved from a Type Ic to a Type IIn over the span of a few years, suggest that not only SNe II but potentially all supernovae may show signatures of interaction with circumstellar material. Consequently, Turatto's revised classification scheme [10] includes a broad category of "interacting SNe" that spans all SN types and includes the SNe IIn.

The IIn category has always been heterogeneous, as noted by Filippenko [2], whose Figure 14 presented a non-coeval collection of SN IIn spectra. Figure 1 shows that even when compared at similar ages, SNe IIn have quite diverse spectral characteristics. The primary feature of $\mathrm{SNe}$ IIn spectra, the strong $\mathrm{H} \alpha$ emission line, also varies substantially in strength and profile between objects of comparable age (Figure 2) and even for a given object over time. In particular, the very narrow (FWHM $<200 \mathrm{~km} / \mathrm{s}$ ) component of $\mathrm{H} \alpha$ 


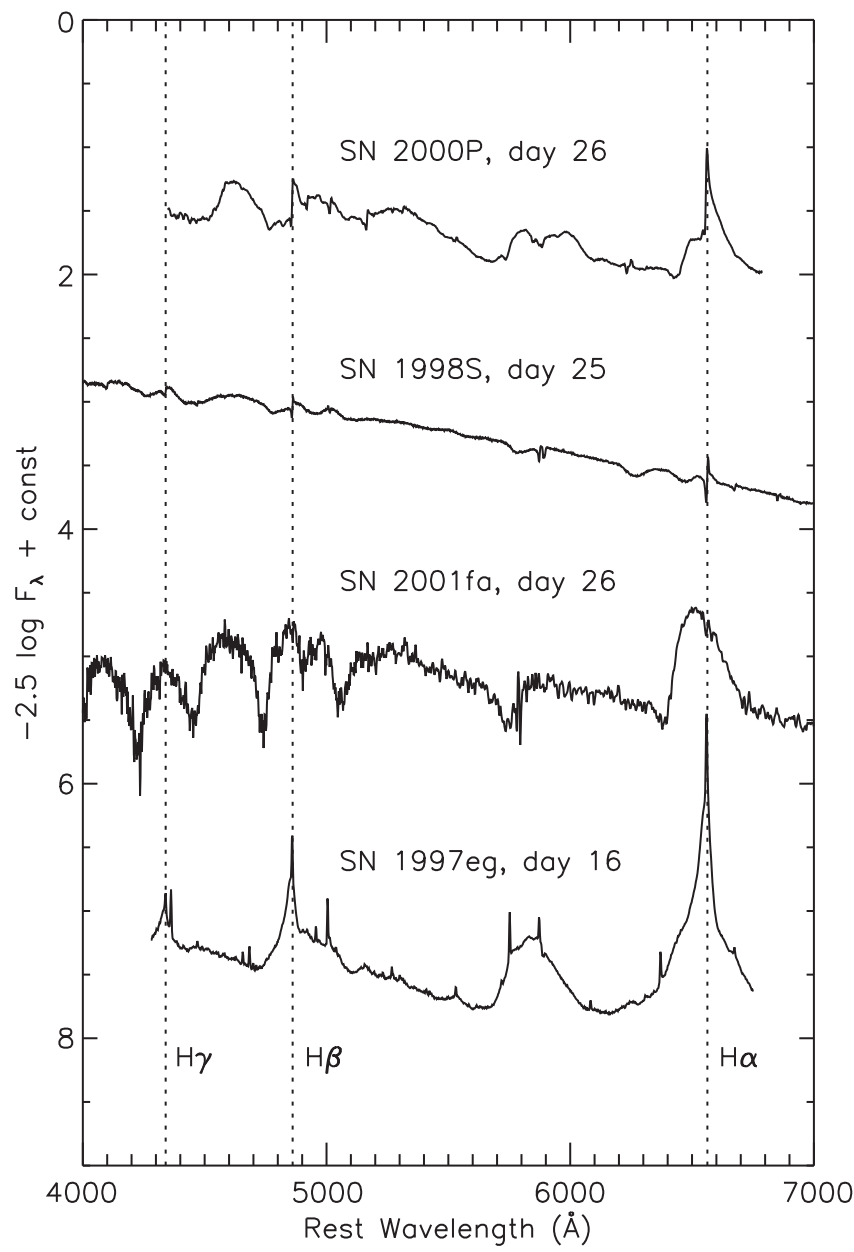

FIGURE 1: Deredshifted optical spectra of four Type IIn supernovae at similar epochs, showing the diversity of spectral characteristics within the subtype. All dates are post-discovery. Detailed analyses of SN 1997eg and SN 2000P have been conducted by [11] and [12], respectively; the other data are presented courtesy of A. Filippenko (priv. comm.). For the unpublished spectra, redshift corrections are based on information in the Asiago Supernova Catalog [13]. Hydrogen Balmer lines are labeled.

does not necessarily exist at all times in the evolution of a SN IIn. In SN 1997eg (Figure 3; [11]), this narrow $\mathrm{H} \alpha$ component disappeared after 100 days post-discovery, was replaced by a small symmetric absorption feature, and then reappeared around day 400 . The H $\alpha$ line in SN 1998S ([12]; their Figure 7) also lost its narrow emission component early on, but was very asymmetric and changed much more dramatically over time.

Because of interaction with circumstellar material, the light curves of SNe IIn often decline quite slowly in comparison with other core-collapse objects, but not all members of the subclass show this slow decline [2]. The canonical IIn SN $1988 \mathrm{Z}$ decreased in brightness by only 5 magnitudes in 1000 days [14], but others decline more quickly (e.g., SN 1998S; [15]). SN 1994W faded by $\sim 6$ magnitudes in $B$ in only 100 days [16].

Circumstellar interaction can also cause some $\mathrm{SNe}$ IIn to become strong radio and X-ray emitters; SN $1988 Z$ and SN 1986J are among the brightest radio supernovae ever observed [17]. Nearly all the SNe IIn detected in X-rays have also been strong radio sources [18, 19]. However, not all SNe IIn become radio-loud [18], and there is considerable heterogeneity even among the "radio-quiet" subset [2]. The overlap between the IIn, II-P, and II-L categories makes it difficult to identify trends in radio brightness with core-collapse subtype. 

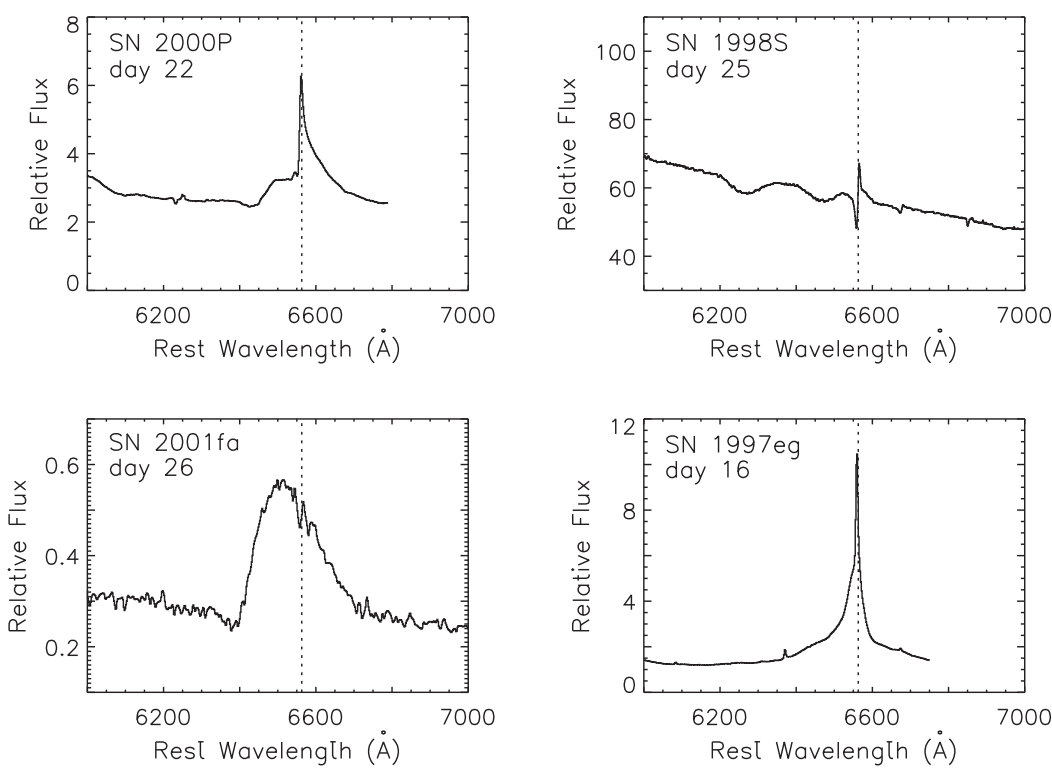

FIGURE 2. As in Fig. 1, but for the $\mathrm{H} \alpha$ region of the spectrum.

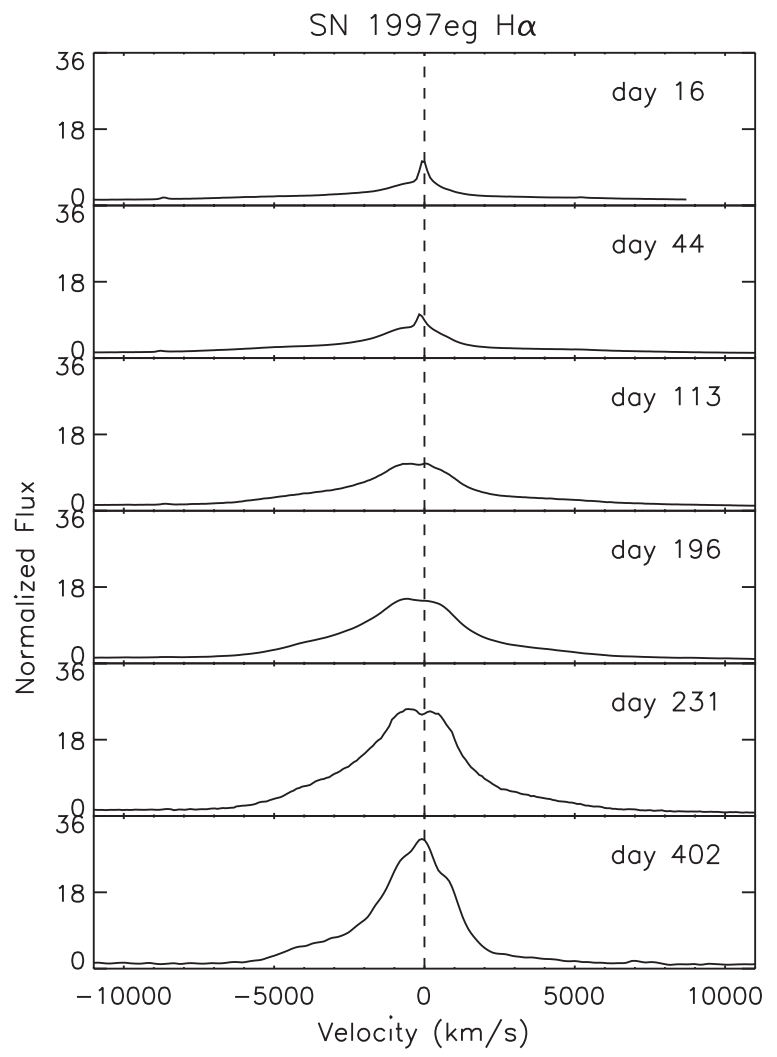

FIGURE 3. Time evolution of the multi-component $\mathrm{H} \alpha$ profile of SN 1997eg [11]. Spectra are normalized to day 16 at $v=-10,000 \mathrm{~km} \mathrm{~s}^{-1}$. 

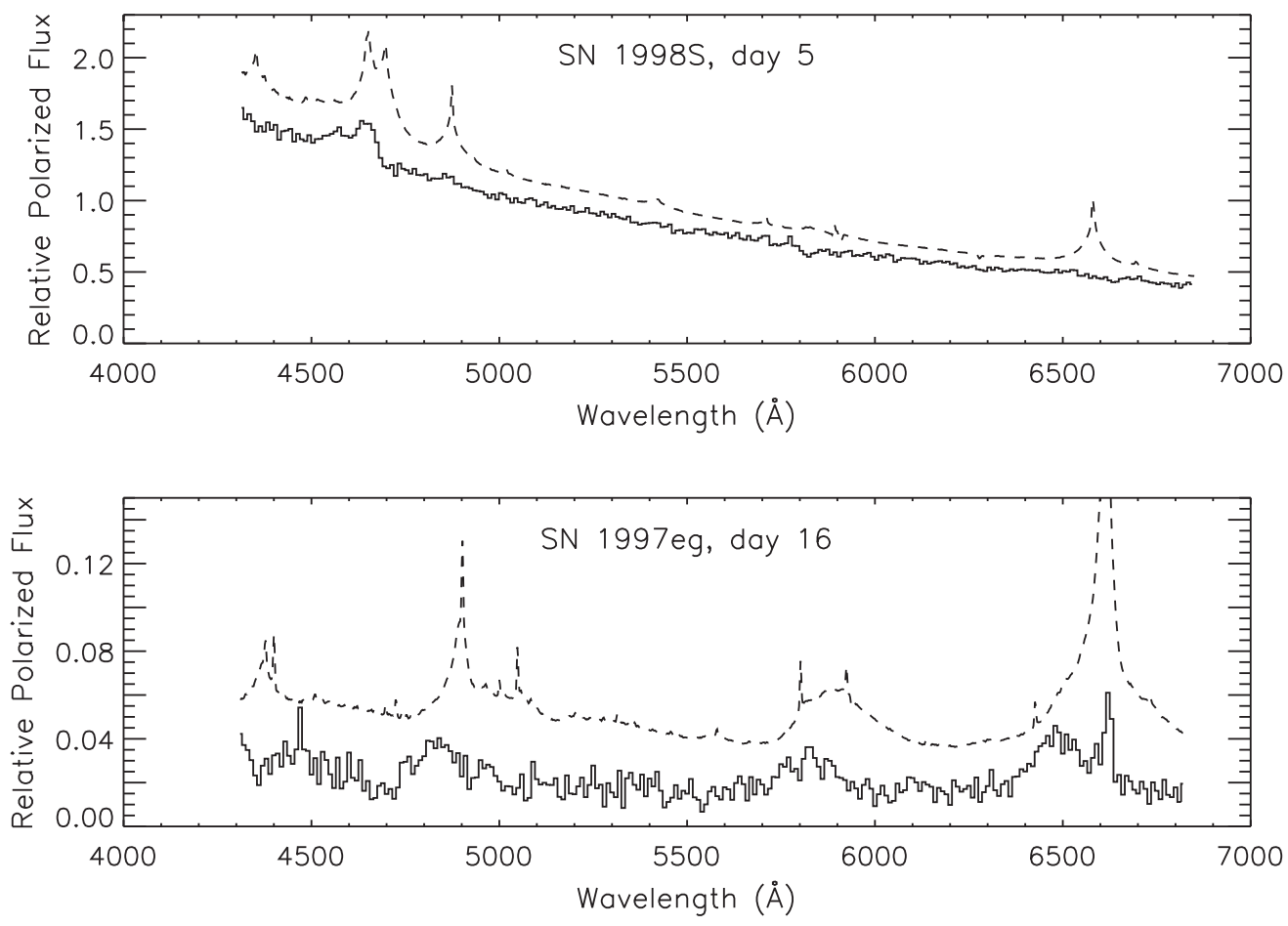

FIGURE 4. Polarized flux spectra (percent polarization multiplied by flux) for SN 1998S [12] and SN 1997eg [11], binned to 10 Angstroms in each case. Dashed lines show total flux spectra for comparison, scaled by 0.037 and 0.036 , respectively. Polarization data are corrected for estimated interstellar polarization (ISP) effects; these estimates are somewhat uncertain in each case, but a simple correction for ISP could not account for all the differences between these two polarized flux spectra.

Finally, SNe IIn show considerable variety in their spectropolarimetric characteristics (Figure 4). Supernovae of all types are known to be polarized due to intrinsic asphericity of the ejecta. The circumstellar material surrounding SNe IIn can produce its own polarization signature in addition to that arising from the ejecta. Some of the polarization variations between SNe IIn are likely due to inclination; that is, they may have similar geometrical distributions of circumstellar material, but have different polarization signatures due to different viewing angles. To study this effect, I am constructing a grid of Monte Carlo radiative transfer models of the $\mathrm{H} \alpha$ line polarization produced by circumstellar matter distributions with various geometries and seen at various viewing angles [20]. Preliminary results suggest that differences in viewing angle may account for some of the polarimetric variety in SNe IIn. However, such geometric effects cannot explain all of the IIn diversity; for example, the transformation of SN 2001em from a Ic into a IIn [8, 9] is very unlikely to be due to a sudden change in inclination!

These examples show that the IIn classification is currently something of a "catchall" for a number of related but distinct objects, all with close connections to Turatto's [10] new category of "interacting SNe". Now that circumstellar interaction has been recognized to occur for a broad range of supernova types, and now that high-quality time-dependent data are more readily available, we are in a good position to reconsider the categorization of SNe IIn and other interacting supernovae. An initiative underway 
within the Berkeley supernova group will quantify the spectral and spectropolarimetric characteristics of SNe IIn and search for correlations between these features and other properties such as light curve shape and radio/X-ray behavior. If found, such correlations will illuminate the relationships between diverse members of the IIn category and perhaps ultimately argue for the category's subdivision.

In the future, full understanding and correct classification of these important objects will depend critically on obtaining a broad range of observational information, including multiwavelength and polarimetric data, with as much time coverage as possible. In addition, supernova researchers should expand their collaborations with the evolved star community. If the circumstellar media around SNe IIn and related objects are indeed created by winds and outflows from massive stars, we can use nearby evolved star nebulae as analogous cases to the circumstellar envelopes of supernovae. Such an effort could greatly improve our understanding of supernova progenitors and help shed light on the physical causes of the diversity we observe among interacting supernovae.

\section{ACKNOWLEDGMENTS}

This research was funded by an NSF Astronomy \& Astrophysics Postdoctoral Fellowship, AST-0302123; by NSF grant AST-0607485 to A. V. Filippenko; and by the National Energy Research Scientific Computing Center, US DOE Contract \#DE-AC0376SF00098. I thank Alex Filippenko at UC Berkeley and Peter Nugent at the Lawrence Berkeley Laboratory for their support and invaluable contributions.

\section{REFERENCES}

1. E. M. Schlegel, MNRAS 244, 269 (1990).

2. A. V. Filippenko, ARAA 35, 309 (1997).

3. E. Cappellaro, M. Turatto, D. Y. Tsvetkov, et al., A\&A 322, 431 (1997).

4. M. Turatto, S. Benetti, and E. Cappellaro, "Variety in Supernovae," in From Twilight to Highlight: The Physics of Supernovae, Springer, Berlin, 2003, p. 200.

5. J. Deng, K. S. Kawabata, Y. Ohyama, et al., ApJ 605, L37 (2004).

6. M. Hamuy, M. M. Phillips, N. B. Suntzeff, et al., Nature 424, 651 (2003).

7. G. Aldering, P. Antilogus, S. Bailey, et al., ApJ 650, 510 (2006).

8. A. M. Soderberg, A. Gal-Yam, and S. R. Kulkarni, GCN 2586, 1 (2004).

9. N. N. Chugai, and R. A. Chevalier, ApJ 641, 1051-1059 (2006).

10. M. Turatto, "Classification of Supernovae," in This Volume, AIP, New York, 2007.

11. J. L. Hoffman, D. C. Leonard, R. Chornock, et al. (2007), in prep.

12. D. C. Leonard, A. V. Filippenko, A. J. Barth, et al., ApJ 536, 239 (2000).

13. R. Barbon, V. Buondí, E. Cappellaro, et al., A\&AS 139, 531 (1999).

14. M. Turatto, E. Cappellaro, I. J. Danziger, et al., MNRAS 262, 128 (1993).

15. A. Fassia, W. P. S. Meikle, W. D. Vacca, et al., MNRAS 318, 1093 (2000).

16. R. J. Cumming, and P. Lundqvist, "Supernova Progenitor Constraints from Circumstellar Interaction: Type II," in Advances in Stellar Evolution, Cambridge University Press, Cambridge, 1997, p. 297.

17. R. A. Chevalier, C. Fransson, and T. K. Nymark, ApJ 641, 1029 (2006).

18. S. D. van Dyk, M. Hamuy, and A. V. Filippenko, AJ 111, 2017 (1996).

19. D. Pooley, W. H. G. Lewin, D. W. Fox, et al., ApJ 572, 932 (2002).

20. J. L. Hoffman, "Polarized Line Profiles as Diagnostics of Circumstellar Geometry in Type IIn Supernovae," in Circumstellar Media and Late Stages of Massive Stellar Evolution, Rev. Mex. AA Ser. Conf., 2007, in press. 